\title{
The Natural Law of Transition of a Charged Particle into a Compound State under the Action of an Electroscalar Field
}

\author{
Oleg Antonovich Zaimidoroga \\ Joint Institute for Nuclear Research, Dubna, Russian Federation \\ Email: zaimidor@jinr.ru
}

How to cite this paper: Zaimidoroga, O.A. (2016) The Natural Law of Transition of a Charged Particle into a Compound State under the Action of an Electroscalar Field. Journal of Modern Physics, 7, 2188-2204. http://dx.doi.org/10.4236/jmp.2016.715190

Received: August 23, 2016

Accepted: November 22, 2016

Published: November 25, 2016

Copyright $\odot 2016$ by author and Scientific Research Publishing Inc. This work is licensed under the Creative Commons Attribution International License (CC BY 4.0).

http://creativecommons.org/licenses/by/4.0/

\begin{abstract}
This article is the continuation of article [1] where the experimental facts of observation of the electroscalar radiation in the spectrum of the Sun have been presented [2]. This radiation comes into the world having a long wavelength, being longitudinal and extraordinarily penetrating. In accordance with the principle of least action, the Lagrangian of the electroscalar field and the tensor of energy-moment are determined using the variation the potential and coordinates. The equation of motion the charged particle in electroscalar field is determined and the energy of particle has the negative sign with respect to the mechanical energy of particle and the energy of electromagnetic field. So, this is decreasing the electrical potential of particle during the propagation. The electroscalar energy of charged particle and field's force acting on the particle during their motion change the particle's electrical status which, in its turn, may trigger the transition of the particle into a compound state during interaction with any object. Due to the continuity this process can lead the particle to the state which enters into a compound state with a negative energy for a different particle's velocity. This state is the physical vacuum's state. Analysis of the solar spectrum demonstrates that scattering and absorption of electroscalar wave go on the cavities of solids. The spreading out of electroscalar field obeys to the law of plane wave and the transfer the energy and information can occur in vacuum and any medium.
\end{abstract}

\section{Keywords}

Equation of Motion of Electroscalar Field, Tensor Energy-Moment of Electroscalar Field, Natural Law of Particle's Transition into a Compound State, Physical Vacuum

\section{Introduction}

Present article is the continuation of article [1] where the experimental facts of 
observation the electroscalar radiation in the spectrum of the Sun have been presented. This radiation comes into the world having a long wavelength, being longitudinal and extraordinarily penetrating. One of the way to create the theory of this phenomenon it was using the analogy between the linear theory of elasticity and electrodynamics. It's known that theory of elasticity [3] [4] contents both transverse and longitudional components of the vector of displacement in the equation of motion of elastic wave. So, from the transversal component of the vector of displacements the Maxwell equation has been resulted, and from the longitudinal component of the vector displacements the equation of electroscalar field has been received [2]. Moreover, the four-vector Maxwell electromagnetic potential and four-scalar potential neither form a single object in the Minkowski space nor interfere and, as a consequence, prove to be independent [5] [6]. Spectrum of radiation has a discrete character and single radiation signal posseses an exceedingly small value of raising time of electrical signal of front and drop of the order of fractions of a picosecond. Average amplitude of spectrum has a negative value. A 2-D analysis of spectrum shows that solar electroscalar radiation scatters and absorbs on the cavity of sensor made from the copper. Each cavities irradiate a corresponding electroscalar waves. The ability to detect the amplitude and frequency of this radiation would allow to observe an internal structure of the object. The spreading out of electroscalar field obeys to the law of plane wave and the transfer the energy and information can occur in vacuum and any medium. Both field components in the wave in vacuum are directed along the wave propagation being equal in absolute magnitude while in solids electrical component is opposite the electroscalar in forward direction. The equation of motion the charged particle in electroscalar field is determined that the energy of particle has the negative sign with respect to the mechanical energy and to the energy of electromagnetic field. The energy of charged particle in electroscalar field and field force acting on the particle change the electrical status of particle which causes the particle's transition into a compound state during the contact with any object. This state is the state of a PHYSICAL VACUUM. A single particle moves in electroscalar field will permanently and decrease the electrical potential and mass and finaly lost their individual properties. So, the continuity of this process may lead to a practicaly neutral state having however an electroscalar component but electrical component is not negligible. In articles [7] [8] [9] were shown that laws of conservation of the energy and charge in vacuum state are fulfilled.

\section{Generalized Electrodynamics}

The analogy with linear theory of elasticity, supporting propagation of both longitudional and transcendental waves, will give a strictly indication of how the basic equations of generalized electrodynamics should look like. Thus due to this the equation for the Maxwell electromagnetic field was derived from the transcendental component of the displacement vector, and an equation for the electroscalar field from the longitudinal component of the displacement vector. The continuity equation of the electromagnetic field contains displacement of current, and the electroscalar field 
contains displacement of charge. In the continuous medium the system takes the form for the Maxwell equation:

$$
\begin{gathered}
-\frac{1}{c} \frac{\partial \mathbf{E}_{\perp}}{\partial t}+\operatorname{rot} \mathbf{H}=0, \\
\frac{1}{c} \frac{\partial \mathbf{H}}{\partial t}+\operatorname{rot} \mathbf{E}_{\perp}=0, \\
\operatorname{div} \mathbf{H}=0,
\end{gathered}
$$

and another for the electroscalar fields $\mathbf{E}_{\|}$and $W$ are:

$$
\begin{gathered}
\frac{1}{c} \frac{\partial \boldsymbol{E}_{\|}}{\partial t}+\nabla W=0, \\
\frac{1}{c} \frac{\partial W}{\partial t}+\operatorname{div} \boldsymbol{E}_{\|}=0, \\
\operatorname{rot} \boldsymbol{E}_{\|}=0 .
\end{gathered}
$$

Such a system describes propagation of longitudinal waves in a continuous medium. Because the transverse and longitudinal waves propagate in the elastic continuum with different velocities, $c_{t}$ for the transverse and $c_{l}$ for the longitudinal ones, the fields $\mathbf{E}_{\perp}$ and $\mathbf{E}_{\|}$can be considered as independent. The velocities of propagation of the longitudinal and transverse waves will be the same in this article. Let us introduce the following field definitions:

$$
\begin{gathered}
\mathbf{E}_{\perp}=-\frac{1}{c} \frac{\partial \mathbf{A}}{\partial t}, \quad \mathbf{H}=\operatorname{rot} \mathbf{A} \\
\mathbf{E}_{\|}=\nabla \lambda, \quad W=-\frac{1}{c} \frac{\partial \lambda}{\partial t},
\end{gathered}
$$

where $\mathbf{A}$ are the time and space parts of the electromagnetic 4-potential. It should be noted here that by virtue of their definition the values $\mathbf{E}_{\|}$and $W$ are the components of a 4 -vector in the Minkowski space-time.

\section{An Absorption of the Electroscalar Radiation and a Plane Electroscalar Wave}

The electroscalar radiation is produced by the structures of solar plasma providing a spectrum with a sign-alternating amplitude of order of 20 millivolt and a frequency (by Fourier) up to 500 Hertz. The averaged value of the spectrum is negative and equals minus 2 - 5 millivolt, with a positive-amplitude signal coming on every millisecond and a negative-amplitude signal following after an interval. The frequency analysis of the spectrum shows that this radiation has a long wavelength and a practically constant amplitude. A single radiation signal has an exceedingly small value of raising time of electrical signal of front and drop of the order of fractions of a picosecond.

The spectrum of the solar electroscalar radiation is given in Figure 1.

Positive signal is the electrical potential applying along line of spreading out and contributing to the repulsion of charges due to their polarization. Negative signal is the result of comression charges by rotation the electrical field on the contour of surface 


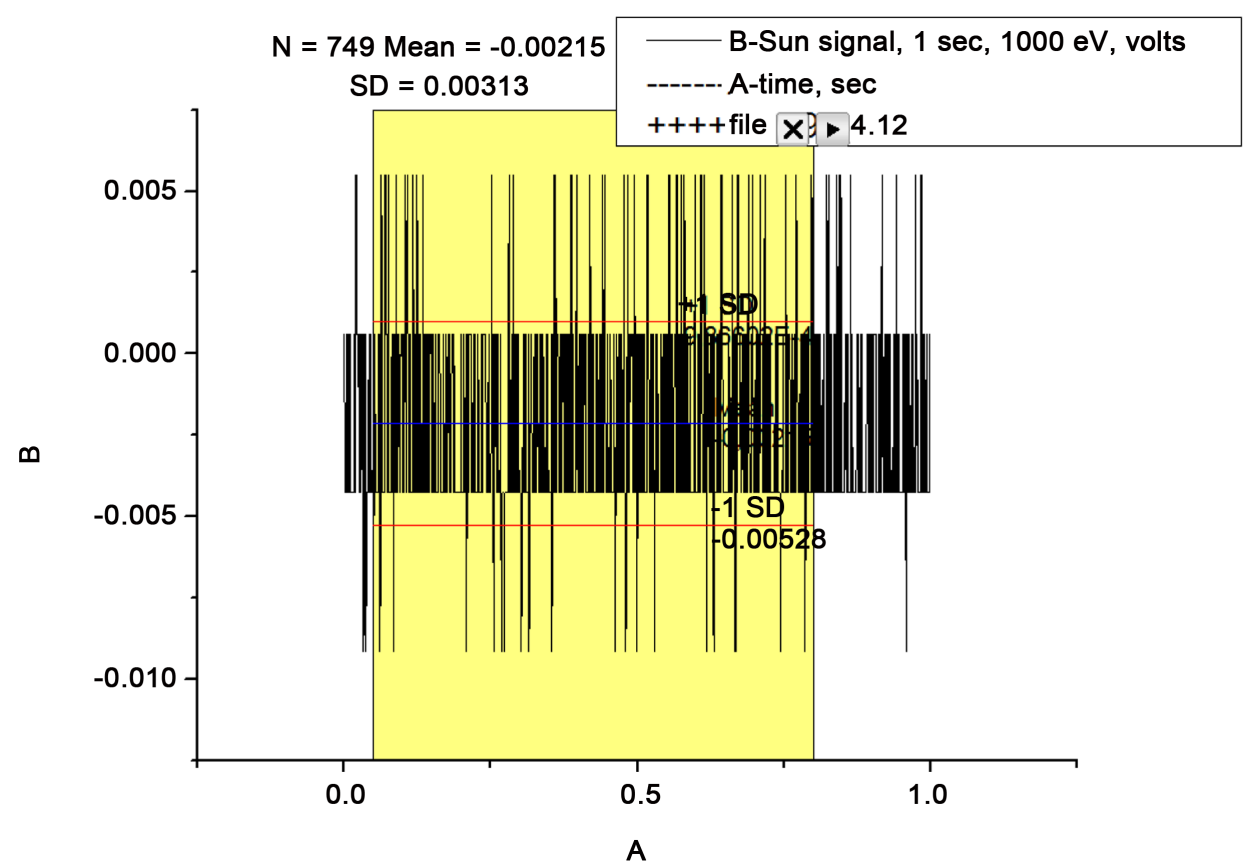

Figure 1. Spectrum of the Sun electroscalar radiaton.

surrounded the volume of charges. In solid states the electroscalar radiation losses their energy on the cavities and due to that reradiate corresponding plasmons electroscalar signal [10]. The amplitude of signal in observed spectrum tells about presence in the metall of sensor varies cavities. In the case when the signal corresponds to the frequency of cavity may appear the enhancement the outgoing electroscalar signal (plasmon resonanse). Therefore, this radiation can be used to study the structure of objects, which is similar examination using x-rays. On the Figure 2 the 2-D graph of electroscalar signal of the Sun taken by $\mathrm{Cu}$-spherical sensor is presented. For this aim the amplitude of signal $B_{i}$ presented as $\delta B_{i}=B_{i+1}-B_{i}$. Two projection of axis is components of signal $B_{i}$.

The spectrum of the Solar electroscalar radiation may be presented as a plane electroscalar wave. Particularly, the second equation of the electroscalar field is such that the electric field $\mathbf{E}_{\|}$is expressed in terms of the scalar potential $\lambda$ :

$$
\operatorname{div} \mathbf{E}_{\|}+\frac{1}{c} \frac{\partial \mathbf{W}}{\partial t}=-4 \pi \rho_{\|} .
$$

Since $\mathbf{E}_{\|}=\nabla \lambda$ and $\mathbf{W}=-\frac{1}{c} \frac{\partial \lambda}{\partial t}$, the equation that is satisfied by the potential of the electroscalar field in matter is:

$$
\Delta \lambda=-4 \pi \rho_{\|}+\frac{1^{2}}{c} \frac{\partial^{2} \lambda}{\partial t^{2}}
$$

From this equation it follows that the potential of the electroscalar field may have maxima and minima as the field in vacuum differs from the Laplace equation for the electromagnetic field with a zero right side and second derivatives have equal signs.

In vacuum, with $\rho_{\|}=0$, this equation may be given as: 


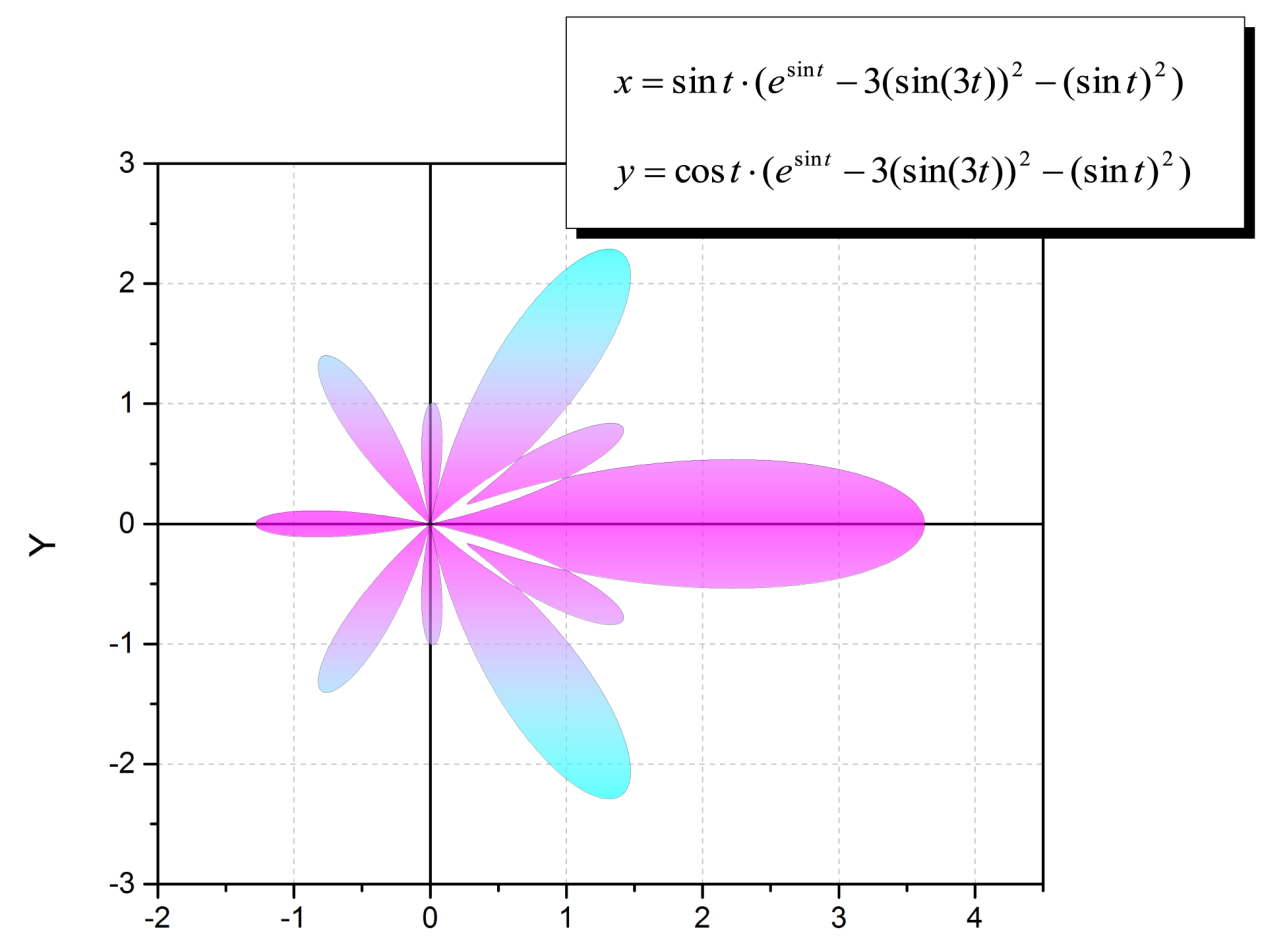

Figure 2. 2-D graph of cavities.

$$
\frac{\partial^{2} \lambda}{\partial t^{2}}-c^{2} \frac{\partial^{2} \lambda}{\partial x^{2}}=0 .
$$

In order to find a solution for this equation, we will present it in the form:

$$
\left[\left(\frac{\partial}{\partial t}-c \frac{\partial}{\partial x}\right)\left(\frac{\partial}{\partial t}+c \frac{\partial}{\partial x}\right)\right] \lambda=0
$$

The equation for the plane wave has the following solution:

$$
\Lambda=\lambda_{1}\left(t-\frac{x}{c}\right)+\lambda_{2}\left(t+\frac{x}{c}\right),
$$

which describes two waves travelling in the direction $+X$ :

$$
\lambda_{1}\left(t-\frac{x}{c}\right)
$$

as well as in the direction $-X$ :

$$
\lambda_{2}\left(t+\frac{x}{c}\right)
$$

When $t-\frac{x}{c}=$ const, the field changes with the time and at each moment it is different for different $\mathbf{X}$. The electroscalar field has the same amplitude for the coordinates satisfying the relation $t-\frac{x}{c}=$ const for the $+\mathbf{X}$ direction. If the field has a particular value at some point, then in the time $t$ it will take the same value at a point 
being at the distance $c t$ from the previous place. This implies that for all the values of the electroscalar field it propagates in space with the velocity ${ }^{\star} c^{\star}$. This will be in a similar way for the $\mathbf{- X}$ direction. We will now express the plane wave equation for the electroscalar field in terms of the field components $\mathbf{W}$ and $\boldsymbol{E}_{\|}$:

$$
\mathbf{E}_{\|}^{2}-\mathbf{W}^{2}=0
$$

i.e. $|\mathbf{E}|_{\|}=|\mathbf{W}|$, both field components in the plane wave in vacuum are directed along the wave propagation being equal in absolute magnitude. The energy flux in a plane wave, i.e. the Pointing vector, will be:

$$
\mathbf{S}=\frac{c}{4 \pi} \mathbf{E}_{\|} \mathbf{W} \times n=\frac{c}{4 \pi} \mathbf{E}_{\|}^{2}=\frac{c}{4 \pi} \mathbf{W}^{2},
$$

where $n$ is a unit vector along the direction of the electroscalar wave propagation. Correspondingly, the energy of the wave will be as follows:

$$
\mathbf{E}=\frac{\mathbf{E}_{\|}^{2}+\mathbf{W}^{2}}{8 \pi}=\frac{\mathbf{E}_{\|}^{2}}{4 \pi}=\frac{\mathbf{W}^{2}}{4 \pi} .
$$

The flux of the field pulse is defined by the value of the field energy flux density. And the absolute value of the quantities $|\mathbf{E}|_{\|}$and $|\mathbf{W}|$ transforms as

$$
\sqrt{\mathbf{E}}
$$

For the electromagnetic field the potentials can be chosen in such a way that $\phi=0$, with $\operatorname{div} \mathbf{A}=0$, then the electric and magnetic fields of the plane wave are perpendicular to one another and are equal in absolute magnitude.

\section{Lagrangian of the Longitudinal Electroscalar Field}

The properties of a charged particle relating to its interactions with the electroscalar field are defined by one parameter: that is the particle charge which can be both positive and negative. The properties of the electroscalar field are characterized by the four-dimensional scalar potential $\lambda$ which is the coordinate and time function. The quantity $\lambda$ comes into action in accordance with the principle of least action as:

$$
\frac{e}{c} \int_{a}^{b} \lambda \mathrm{d} s,
$$

where the integral is taken at the points a to $b$ of the world line of a particle. The type of action can be established here on the grounds that the Lagrangian is a scalar, while the product of the charge and potential is the particle's potential energy. Besides that, the relativistic invariance requirement allows for the scalar function the form:

$$
\int_{a}^{b} \lambda \mathrm{d} s
$$

Thus, the action for a charge in the electroscalar field appears as:

$$
\mathbf{S}=\int_{a}^{b}\left(-m c-\frac{e}{c} \lambda\right) \mathrm{d} s,
$$

where the 1 st term is the energy of a free particle, and the 2 nd term is the potential 
energy of the particle. According to the principle of longitudinal superposition, the charge may be expressed in terms of the charge density:

$$
\rho_{\|}=\sum e \delta\left(r_{i+1}-r_{i}\right)
$$

In such a case, summation goes over all the linear charges.

The action can be represented as a time integral:

$$
\mathbf{S}=\int_{a}^{b} L \mathrm{~d} t
$$

where $L$ is the Lagrange function.

Since $\mathrm{d} s=c \sqrt{1-\frac{v^{2}}{c^{2}}} \mathrm{~d} t$, the action of the electroscalar field will be as follows:

$$
\mathbf{S}=\int_{a}^{b}\left(-m c^{2} \sqrt{1-\frac{v^{2}}{c^{2}}}-e \lambda \sqrt{1-\frac{v^{2}}{c^{2}}}\right) \mathrm{d} t
$$

So, the Lagrange function of the electroscalar field is:

$$
\mathbf{L}=-m c^{2} \sqrt{1-\frac{v^{2}}{c^{2}}}-e \lambda \sqrt{1-\frac{v^{2}}{c^{2}}}
$$

In the non-relativistic case, $\mathbf{L}=m v^{2} / 2-\frac{e}{c} \lambda$.

\section{The Energy-Momentum Tensor of the Electroscalar Field}

In order to determine the energy-momentum tensor of the electroscalar field and establish the equation of motion of a particle in the four-dimensional form, we apply the principle of least action in the relativistic case. The variables which vary in a principle of least action are field potentials and coordinates of the world line. The action of a charge in the electroscalar field in the three-dimensional form is as follows:

$$
\mathbf{S}=\int\left(-m c-\frac{e}{c} \lambda\right) \mathrm{d} s
$$

The principle of least action states:

$$
\delta \mathbf{S}=\delta\left(\int\left(-m c-\frac{e}{c} \lambda\right) \mathrm{d} s\right)
$$

and since: $\mathrm{d} s^{2}=-\left(\mathrm{d} x^{2}+\mathrm{d} y^{2}+\mathrm{d} z^{2}-\mathrm{d} t^{2}\right)=-\mathrm{d} x_{i}^{2}$, the variation of action in the four-dimensional form is:

$$
\delta \mathbf{S}=\int\left[-m c \delta\left(\sqrt{-\mathrm{d} x_{i}^{2}}\right)-\frac{e}{c} \delta\left(\lambda \sqrt{-\mathrm{d} x_{i}^{2}}\right)\right]=0
$$

The action variation takes place along the world line from $a$ to $b$.

Further:

$$
\begin{gathered}
\delta \mathbf{S}=\int\left(m c \frac{\mathrm{d} x_{i}}{\mathrm{~d} s} \delta \mathrm{d} x_{i}-\frac{e}{c} \delta(\lambda) \mathrm{d} s+\frac{e}{c} \lambda \frac{\mathrm{d} x_{i}}{\mathrm{~d} s} \delta \mathrm{d} x_{i}\right)=0, \\
\delta \mathbf{S}=\int\left(m c u_{i} \mathrm{~d} \delta x_{i}-\frac{e}{c} \delta(\lambda) \mathrm{d} s+\frac{e}{c} \lambda \mathbf{u}_{i} \mathrm{~d} \delta x_{i}\right)=0
\end{gathered}
$$


In the first and third terms we integrate by parts; differentiation of $\delta x_{i}$ and $\mathrm{d} x_{i}$ here can be interchanged, $\boldsymbol{u}_{i}=\frac{\mathrm{d} x_{i}}{\mathrm{~d} s}$ is the four-velocity of the particle.Thus, we obtain:

$$
\delta \mathbf{S}=\left[\left.m c \mathbf{u}_{i} \delta x_{i}\right|_{a} ^{b}+\left.\frac{e}{c} \mathbf{u}_{i} \lambda \delta x_{i}\right|_{a} ^{b}\right]+\int\left[-m c \mathrm{~d} \mathbf{u}_{i} \delta x_{i}-\frac{e}{c} \delta(\lambda) \mathrm{d} s-\frac{e}{c} \mathrm{~d}\left(\lambda \mathbf{u}_{i}\right) \delta x_{i}\right]=0
$$

The expressions in the square brackets are equal to zero because in the limits $\left.\delta x_{i}\right|_{a}$ and $\left.\delta x_{i}\right|^{b}$ they equal zero due to the zero field at infinity. Next, the expression for variation takes the form:

$$
\begin{gathered}
\left.m c \mathbf{u}_{i} \delta x_{i}\right|_{a} ^{b}+\left.\frac{e}{c} \mathbf{u}_{i} \lambda \delta x_{i}\right|_{a} ^{b}=0 \\
\delta S=\int\left[-m c \mathrm{~d} \mathbf{u}_{i} \delta x_{i}-\frac{e}{c} \delta(\lambda) \mathrm{d} s-\frac{e}{c}\left(\mathrm{~d}(\lambda) \mathbf{u}_{i} \delta x_{i}+\mathrm{d} u_{i} \lambda \delta x_{i}\right)\right]=0
\end{gathered}
$$

Since

$$
\mathrm{d} \mathbf{u}_{i}=\frac{\mathrm{d} u_{i}}{\mathrm{~d} s} \mathrm{~d} s, \quad \delta \lambda=\frac{\partial \lambda}{\partial x_{i}} \delta x_{i}, \quad \mathrm{~d} \lambda=\frac{\partial \lambda}{\partial x_{i}} \mathrm{~d} x_{i}, \quad u_{i} u_{i}=-1 .
$$

The expression for variation takes the form:

$$
\delta \mathbf{S}=\int\left[-\left(m c+\frac{e \lambda}{c}\right) \mathrm{d} u_{i} \delta x_{i}-\frac{e}{c} \frac{\partial \lambda}{\partial x_{i}} \delta x_{i} \frac{\mathrm{d} x_{k}}{u_{k}}-\frac{\partial \lambda}{\partial x_{k}} \mathrm{~d} x_{k} u_{i} \delta x_{i}\right]=0 .
$$

Besides, $\mathrm{d} x_{i}=u_{i} \mathrm{~d} s$. Here, the $i$ and $k$ indices can be interchanged due to summation. Thus, we obtain:

$$
\delta \mathbf{S}=\int-\left(m c+\frac{e \lambda}{c}\right) \mathrm{d} u_{i} \delta x_{i}-\frac{e}{c}\left[\frac{\partial \lambda}{\partial x_{i}} \frac{\mathrm{d} x_{k}}{u_{k}} \delta x_{i}+\frac{\partial \lambda}{\partial x_{i}} u_{i} \mathrm{~d} x_{k} \delta x_{i}\right]=0 .
$$

The expression takes thes form:

$$
\delta \mathbf{S}=\int-\left(m c+\frac{e \lambda}{c}\right) \mathrm{d} u_{i} \delta x_{i}-\frac{e}{c}\left[\frac{-\partial \lambda}{\partial x_{i}} u_{k} u^{k} \mathrm{~d} s \delta x_{i}+\frac{\partial \lambda}{\partial x_{k}} u_{k} \mathrm{~d} s u_{i} \delta x_{i}\right]=0
$$

From the arbitrariness of $\delta x_{i}$ it follows that the integrand is equal to zero and the equation of motion of a particle moving in an electroscalar field thus has the form:

$$
\left(m c+\frac{e \lambda}{c}\right) \frac{\mathrm{d} u_{i}}{\mathrm{~d} s}=-\frac{e}{c} Z_{i k} u_{k}
$$

where

$$
Z_{i k}=\left(\frac{\partial \lambda}{\partial x_{i}} u_{k}-\frac{\partial \lambda}{\partial x_{k}} u_{i}\right)
$$

is the 4-dimential antisymmetric tensor of 2 -nd order:

$$
Z_{i i}=0, Z_{i k}=-Z_{k i}, Z_{i k}=\left(\nabla_{i} \lambda \frac{\partial \lambda}{\partial t}\right)=\left(E_{\|},-W\right), i, k=1, \cdots, 4 .
$$

The tensor elements are: $Z_{12}=-Z_{21}=\frac{v E_{\|}}{c \sqrt{1-v^{2} / c^{2}}}$, while $Z_{13}=-Z_{31}=\frac{v E_{\|}}{c \sqrt{1-v^{2} / c^{2}}}$ 
Thus, we have obtained the equations for a particle moving in an electroscalar field.

By using the action variation expression in the case when at least one of the limits is not equal to zero, for instance, $\delta x_{i}$, we derive:

$$
\delta S=\left(m c u_{i}+\frac{\rho}{c} u_{i} \lambda\right) \delta x_{i}
$$

Note that $\rho \mathbf{u}_{i}=j_{i}$.

Let us determine now the generalized momentum $\mathbf{P}_{i}=\frac{\partial S}{\partial x_{i}}=p_{i}+j_{i} \frac{\lambda}{c}$

From here we obtain the Hamilton-Jacobi equation:

$$
\left(\frac{\partial S}{\partial x_{i}}-j_{i} \frac{\lambda}{c}\right)^{2}+m^{2} c^{2}=0 .
$$

By the way the equation of motion of a particle moving in an electromagnetic field thus has the form:

$$
m c \frac{\mathrm{d} u_{i}}{\mathrm{~d} s}=\frac{\rho}{C} \mathbf{F}_{i k} u_{k},
$$

Here $F_{\mu v}^{\perp}$ is the electromagnetic tensor in the Coulomb gauge:

$$
\mathbf{F}_{\mu \nu}^{\perp}=\frac{\partial A_{\nu}^{\perp}}{\partial x^{\mu}}-\frac{\partial A_{\mu}^{\perp}}{\partial x^{\nu}}, \quad \phi=0, \quad \operatorname{div} \mathbf{A}^{\perp}=0 .
$$

So we obtain the tensor presenting the covariant law of motion of a charged particle in transverse electromagnetic and longitudinal electroscalar fields:

$$
\mathbf{O}_{\mu v} u^{v}=\mathbf{F}_{\mu v}^{\perp}-\mathcal{Z}_{\mu v}
$$

can be considered as a relativistic law of summation of electromagnetic and electroscalar fields or as a generalized intensity. So the mechanical and electroscalar energis enter to the total energy of charged particle with a different sign. However the electromagnetic energy do not change the mechanical one. This properties may signify that a charged particle could be ready to come into a compound system. The expression for action covers both the action of free charges and their action in the electroscalar field. The action that is depending on the properties of the field itself, without charges, is governed by the field structure and components. Such a term is represented by one of the invariants of the electroscalar field which is defined by the expression $\left(\mathbf{W v}^{2}-\mathbf{c E}_{\|}^{2}\right)$. In the four-dimensional form this invariant has the following appearance: $Z_{i k}^{2}$. From the invariance of this variable it follows that there may be such states for which if $\mathbf{E}_{\|}>\mathbf{W v}$ or $\mathbf{c E}_{\|}<\mathbf{v W}$, then any system having a small component will remain always small and its radiation will be either purely longitudinal electric or longitudinal scalar. 


\section{Transformation Properties of Electroscalar Fields}

From the system of Equations (1) and (5) it follows that longitudinal electroscalar waves are responsible for the transport of Coulomb field. In point of fact, the system of Equation (5) contains an equation for electrostatics $\operatorname{div} \mathbf{E}_{\|}=-4 \pi \rho$ has a solution in the form of longitudinal waves, the time-dependant heterogeneous density of electric charge being the source of these waves. From the definition of three-dimensional fields $\mathbf{E}_{\|}$and $W$ it follows that they are covariant components of the $\lambda$-gradient fourvector:

$$
Z_{i}=\partial_{i} \lambda=\left(\frac{1}{c} \frac{\partial \lambda}{\partial t}, \nabla_{i} \lambda\right)=\left(-W, \mathbf{E}_{\|}\right)
$$

Latin indexes runs from 1 to $4 ; \partial_{i} \lambda=\left(1 / c \partial / \partial t, \nabla_{i}\right)$ are the components of a fourgradient, and $\nabla_{i}$ are the components of a three-gradient. Now we introduce the antisymmetric double tensor in four-dimensional notation:

$$
Z_{i k}=\left(Z_{i} u_{k}-Z_{k} u_{i}\right)
$$

where $u_{i}$ and $u_{k}$ is the 4 -vector of velocity. In the three-dimensional notation the components of this tensor acquire the following form:

$$
\mathcal{Z}_{i k}=\frac{v_{i} W+c E_{\| k}}{\sqrt{c^{2}-v^{2}}}, \quad \mathcal{Z}_{k i}=\frac{-v_{k} W-c E_{\| i}}{\sqrt{c^{2}-v^{2}}} .
$$

Under Lorenz transformations, components of the four-vector $\mathcal{Z}_{\mu}$ are transformed according to the law:

$$
\mathcal{Z}_{\mu}=\Lambda_{\mu}^{v} \mathcal{Z}_{v}
$$

where $\Lambda_{\mu}^{v}$ is the matrix of Lorenz transformations $\Lambda_{\mu}^{v}$ :

$$
\Lambda_{0}^{0}=\frac{1}{\sqrt{1-\frac{V^{2}}{c^{2}}}}, \quad \Lambda_{i}^{0}=\frac{\Lambda_{0}^{0}}{c} V_{i}, \quad \Lambda_{i}^{k}=\delta_{i}^{k}+\left(\Lambda_{0}^{0}-1\right) \frac{V_{i} V^{k}}{V^{2}},
$$

while $V_{i}$ are the components of three-velocity of relative motion of reference frames. So, we obtain:

$$
\begin{gathered}
\mathcal{Z}_{0}^{\prime}=\frac{1}{\sqrt{1-\frac{V^{2}}{c^{2}}}}\left(\mathcal{Z}_{0}+\frac{1}{c}\left(V_{k} \mathcal{Z}_{k}\right)\right) \\
\mathcal{Z}_{i}^{\prime}=\mathcal{Z}_{i}-\frac{V_{i}}{V^{2}}\left(V_{k} \mathcal{Z}_{k}\right)+\frac{1}{\sqrt{1-\frac{V^{2}}{c^{2}}}} \frac{V_{i}}{V^{2}}\left(\left(V_{k} \mathcal{Z}_{k}\right)+\frac{V^{2}}{c} Z_{0}\right),
\end{gathered}
$$

or in the three-dimensional notation

$$
W^{\prime}=\frac{1}{\sqrt{1-\frac{V^{2}}{c^{2}}}}\left(W-\frac{1}{c}\left(\mathbf{V E}_{\|}\right)\right)
$$




$$
\mathbf{E}_{\|}^{\prime}=\mathbf{E}_{\|}-\frac{\mathbf{V}}{V^{2}}\left(\mathbf{V} \mathbf{E}_{\|}\right)+\frac{1}{\sqrt{1-\frac{V^{2}}{c^{2}}}} \frac{\mathbf{V}}{V^{2}}\left(\left(\mathbf{V E}_{\|}\right)-\frac{V^{2}}{c} W\right)
$$

From the definition of the Maxwell electric field:

$$
\mathbf{E}=-\nabla \phi-\frac{1}{c} \frac{\partial \mathbf{A}}{\partial t}
$$

where $\phi$ and $A_{i}$ are the components of electromagnetic four-vector potential, it follows that the field contains both the potential and vortex parts, however, in the Coulomb gauge $\phi=0, \operatorname{div} \mathbf{A}=0$, the Maxwell electric field becomes a vortex one :

$$
\mathbf{E}_{\perp i}=-\frac{1}{c} \frac{\partial \mathbf{A}_{\perp i}}{\partial t} .
$$

Note that the Maxwell field $\mathbf{E}$ can be brought to the vortex type in any inertial reference frame, albeit the Coulomb gauge alone is not Lorenz-invariant [11]. Thus, it is possible to write for the transformation of three-dimensional fields $\mathbf{E}_{\perp}$ and $\mathbf{H}$ the following:

$$
\begin{aligned}
& \mathbf{E}_{\perp}^{\prime}=\frac{\mathbf{V}}{V^{2}}\left(\mathbf{V} \mathbf{E}_{\perp}\right)+\frac{1}{\sqrt{1-\frac{V^{2}}{c^{2}}}}\left(\mathbf{E}_{\perp}-\frac{\mathbf{V}}{V^{2}}\left(\mathbf{V} \mathbf{E}_{\perp}\right)+\frac{1}{c}[\mathbf{V} \times \mathbf{H}]\right), \\
& \mathbf{H}^{\prime}=\frac{V}{V^{2}}(\mathbf{V H})+\frac{1}{\sqrt{1-\frac{V^{2}}{c^{2}}}}\left(\mathbf{H}-\frac{\mathbf{V}}{V^{2}}(\mathbf{V H})-\frac{1}{c}\left[\mathbf{V} \times \mathbf{E}_{\perp}\right]\right) .
\end{aligned}
$$

\section{The Force and the Work of the Electroscalar Field}

We will calculate the generalized momentum for the relativistic Lagrangian (10) of the electroscalar field:

$$
\begin{gathered}
\mathbf{P}=\frac{\partial \mathbf{L}}{\partial v} \\
\mathbf{P}=m_{0} v \frac{1+\frac{e \lambda}{m_{0} c^{2}}}{\sqrt{1-\frac{v^{2}}{c^{2}}}} .
\end{gathered}
$$

The equation of Euler for the in electroscalar field is:

$$
\frac{\mathrm{d} P}{\mathrm{~d} t}=\frac{\partial \mathbf{L}}{\partial r} \text {. }
$$

The time derivative of the momentum vector is the force of the electroscalar field acting on a particle:

$$
\frac{\mathrm{d} P}{\mathrm{~d} t}=\frac{m_{0}}{\sqrt{1-\frac{v^{2}}{c^{2}}}} \frac{\mathrm{d} v}{\mathrm{~d} t}+\frac{e \lambda}{c^{2} \sqrt{1-\frac{v^{2}}{c^{2}}}} \frac{\mathrm{d} v}{\mathrm{~d} t}+\frac{m_{0} v^{2}}{c^{2}\left(1-\frac{v^{2}}{c^{2}}\right)^{\frac{3}{2}}} \frac{\mathrm{d} v}{\mathrm{~d} t}+\frac{e v^{2} \lambda}{c^{4}\left(1-\frac{v^{2}}{c^{2}}\right)^{\frac{3}{2}}} \frac{\mathrm{d} v}{\mathrm{~d} t}+\frac{e v}{c^{2} \sqrt{1-\frac{v^{2}}{c^{2}}}} \frac{\mathrm{d} \lambda}{\mathrm{d} t},
$$

where the full derivative of the four-dimensional quantity $\lambda$ is: 


$$
\frac{\mathrm{d} \lambda}{\mathrm{d} t}=\frac{\partial \lambda}{\partial t}+v \nabla \lambda=v \mathbf{E}_{\|}-c \mathbf{W}
$$

While the velocity varies in its magnitude, the force acting on a particle is directed along the velocity. Further, after simplifications we get:

$$
\frac{\mathrm{d} P}{\mathrm{~d} t}=\frac{m_{0}+\frac{e \lambda}{c^{2}}}{\sqrt{1-\frac{v^{2}}{c^{2}}}}\left(\frac{c^{2}}{c^{2}-v^{2}}\right) \frac{\mathrm{d} v}{\mathrm{~d} t}+\frac{e v}{c^{2} \sqrt{1-\frac{v^{2}}{c^{2}}}}\left(v \mathbf{E}_{\|}-c \mathbf{W}\right) .
$$

The right side of the equation has the appearance:

$$
\begin{gathered}
\frac{\partial \mathbf{L}}{\partial r}=-e \frac{\partial \lambda}{\partial r} \sqrt{1-\frac{v^{2}}{c^{2}}}, \\
\frac{\partial \lambda}{\partial r}=\mathbf{E}_{\|} .
\end{gathered}
$$

It should be pointed out that the derivative of the Lagrangian with respect to the radius is taken at the constant velocity.

Next, we define the force acting on the particle motion in the electroscalar field:

$$
\frac{m_{0}+\frac{e \lambda}{c^{2}}}{\sqrt{1-\frac{v^{2}}{c^{2}}}} \frac{\mathrm{d} v}{\mathrm{~d} t}=\frac{c^{2}-v^{2}}{v^{2}}\left[-\frac{e \lambda}{c^{2} \sqrt{1-\frac{v^{2}}{c^{2}}}}\left(v \mathbf{E}_{\|}-c \mathbf{W}\right)-e \mathbf{E}_{\|} \sqrt{1-\frac{v^{2}}{c^{2}}}\right] .
$$

Thus, the electroscalar force is longitudinal, and its value is $\frac{c^{2}-v^{2}}{v^{2}}$ times larger with regard to the acceleration. The mass of a particle in the electroscalar field varies by the value $\frac{e \lambda}{c^{2}}$. The mass of a particle in the field will be denoted as $\mathbf{m}_{\lambda}=\mathbf{m}_{0}+\frac{e \lambda}{c^{2}}$.

By transforming this expression, we derive:

$$
\frac{m_{\lambda}}{\sqrt{1-\frac{v^{2}}{c^{2}}}} \frac{\mathrm{d} v}{\mathrm{~d} t}=\sqrt{1-\frac{v^{2}}{c^{2}}}\left(\frac{v}{c} e \mathbf{W}-e \mathbf{E}_{\|}\right)
$$

The magnitude of the force $\frac{m_{\lambda}}{\sqrt{1-\frac{v^{2}}{c^{2}}}} \frac{\mathrm{d} v}{\mathrm{~d} t}=\mathbf{F}$ is called the force of the electroscallar field. Finally, we obtain:

$$
\mathbf{F}=-\sqrt{1-\frac{v^{2}}{c^{2}}}\left(e \mathbf{E}_{\|}-\frac{v}{c} e \mathbf{W}\right)
$$

If $\mathbf{E}_{\|}=0$, then the force $\mathbf{F}$ is parallel to the velocity. When $\mathbf{W}=0$, the force $\mathbf{F}$ is directed against the velocity. This is how relativistic transport of the electric field takes place. In the relativistic case, the field moves along the velocity vector, with the electric scalar force $\mathbf{W}$ being directed along the velocity and the electric force, in the opposite direction. It may be seen from the expression for the force that such a 
configuration of fields $\mathbf{E}_{\|}$and $\mathbf{W}$ is preserved at any velocity of motion, and the $\frac{E}{W}$ ratio may be between zero and one. As a next step we will determine the energy of a particle in the electromagnetic and electroscalar fields. According to the definition of energy: $\mathcal{E}=p v-\mathbf{L}$. The energy of the electromagnetic field is then:

$$
\mathcal{E}_{\perp}=\frac{m c^{2}}{\sqrt{1-\frac{v^{2}}{c^{2}}}},
$$

and the particle energy depends on the velocity. The energy of a particle in the electroscalar field equals:

$$
\mathcal{E}_{\|}=\frac{m c^{2}+e \lambda}{\sqrt{1-\frac{v^{2}}{c^{2}}}} .
$$

The electroscalar energy enters into the full energy with a negative value relative to the mechanical energy, while variation of the particle energy with the time is the work done on the particle. In the case of an electromagnetic field, this work is equal to the product of the particle velocity and the force exerted on the particle by the transverse electromagnetic field:

$$
\frac{\mathrm{d} \mathcal{E}_{\perp}}{\mathrm{d} t}=v \frac{\mathrm{d} P}{\mathrm{~d} t}=e \mathcal{E}_{\perp} v
$$

In an electroscalar field this work is the product of the particle velocity and the force acting in the velocity direction:

$$
\frac{\mathrm{d} \mathcal{E}_{\|}}{\mathrm{d} t}=-\mathbf{v} \sqrt{1-\frac{v^{2}}{c^{2}}} e \mathbf{E}_{\|}\left(1-\frac{v}{c} \frac{e \mathbf{W}}{e \mathbf{E}_{\|}}\right) .
$$

Figure 3 illustrates the work on the charged massive particle in electroscalar field for various velocity of particle. Unit of the work is evcm/sec. The energy is 1 mev $($ black $)=4.8 \times 10^{-7}$ egr , $5 \mathrm{mev}($ red $)=2.4 \times 10^{-6}$ erg .

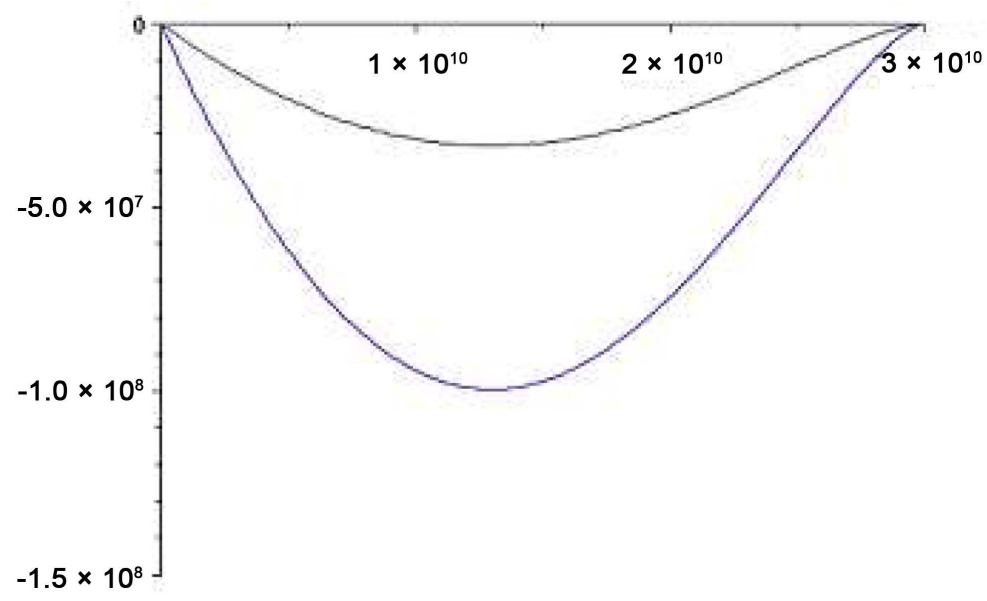

Figure 3. The work on charged particle. 
From this expression it is evident that in the cases when the field $e \mathbf{W v}$ does not exceed $e \mathbf{E}_{\|} c$ at any velocity there is a possibility of the particles transition into compound states.This region is the region where the atomic systems and subsequent elements of structure of mater. The remarkable meaning of this expression for the work done by the electroscalar field on a particle is that the negative energy acquired by the particle changes the electrical status of a charged particle, charged atom or bioparticle. In this event, at lower energies than the energy characteristic for the superposition of particles, an increase the radiation of ${ }^{\star}$ electroscalar photons ${ }^{\star}$ takes place, as well as many acts of particle interactions, attraction and crystallization processes etc. in a medium. Thus, a change in the electric status of the particle provides conditions for a transition of the particle into compound states. For a medium, changes in the particle energy are conditioned by the interaction and attraction to the surrounding objects up to the energy that is characteristic for the longitudinal superposition. Further attraction leads to crystallization in the medium and to fractalization of the structure as well as to the growing repulsion from the object.

\section{The Electron in an Electroscalar Field}

Today, we do not yet comprehend the nature and origins of how various manifestations of the electromagnetic, electroscalar, electroweak types of interaction are combined in one charged particle, for instance, in an electron. The continuity equation of the electroscalar field comprises a displacement charge, which leads to the necessity of taking into account both the field produced by these charges and the field of the charges themselves. Thus, an electron contains a "basic" charge and a displacement charge, which provides enlargement of its size due to the Coulomb law. Now we will try to find the energy of an electron in the electroscalar field. The second equation of the electroscalar field may be written in another form if we employ here the Gauss theorem:

$$
\int_{S} \mathbf{E}_{\|} \mathrm{d} S=-4 \pi \rho_{\|}-\frac{1}{c} \frac{\partial \mathbf{W}}{\partial t} .
$$

The flux of the longitudinal electric field through a spherical surface with the radius $R$ equals:

$$
4 \pi R^{2} \mathbf{E}_{\|}=-4 \pi \rho_{\|}-\frac{1}{c} \rho_{0}, \quad \rho_{0}=\frac{\partial \mathbf{W}}{\partial t} .
$$

We equate the sides and obtain the law of electric intensity (Coulomb law) of the electroscalar field:

$$
\mathbf{E}_{\|}=\left(-\rho_{\|}-\frac{1}{4 c \pi} \rho_{0}\right) \frac{1}{R^{2}}
$$

Thus, the electric field is conditioned both by the charge $e$ and charge displacement $\rho_{0}$; and the radius of the sphere contains two terms, $r_{1}$ and $r_{2}$.

Further, we define the energy of a system of charges proceeding from the expression for the electroscalar field energy density: 


$$
\mathbf{U}=\frac{1}{8 \pi} \int_{V} \mathbf{E}_{\|}^{2} \mathrm{~d} V .
$$

Since $\mathbf{E}_{\|}=\nabla \lambda$, the energy of the field of charges is:

$$
\mathbf{U}=\frac{1}{8 \pi} \int_{V}\left(\mathbf{E}_{\|} \nabla \lambda\right) \mathrm{d} V=\frac{1}{8 \pi} \int_{V}\left(\operatorname{div} \mathbf{E}_{\|} \lambda\right) \mathrm{d} V-\frac{1}{8 \pi} \int_{V}\left(\lambda \operatorname{div} \mathbf{E}_{\|}\right) \mathrm{d} V .
$$

Here, the first integral by the Gauss theorem equals the integral taken through the surface that limits the volume, and the field equals zero at infinity. The second equation of the electroscalar field is substituted into the second integral, and the energy expression for the electroscalar field of a system of charges assumes the form:

$$
\mathbf{U}=\frac{1}{2} \lambda\left(\rho_{\|}+\frac{1}{4 \pi \mathrm{c}} \rho_{0}\right)
$$

If this formula is used for one charge, we can obtain that for $\rho_{\|}=e$ the potential self-energy of the charge (for an electron) is:

$$
\mathbf{U}=\frac{1}{2} \lambda\left(e+\frac{1}{4 \pi c} \rho_{0}\right) .
$$

Thus, an electron contains a "basic" charge and a displacement charge, which provides enlargement of its size due to the Coulomb law. During the electron's motion, the work done by the electroscalar field on the electron discloses the physical meaning of its negative energy and losing its electric status. So, a change in the electric status of the electron depends on the electroscalar energy and provides conditions for its transition into a compound state. If there is no appropriate object to get into a compound state with, the electron can continue to lower its electric status and can be practically neutral with a small mass.

\section{Conclusions}

The following conclusions can be made from this study:

1) A remarkable characteristic of the electrodynamics of electroscalar and electromagnetic fields is that one and the same charged particle may exhibit fairly different properties.

2) The continuity equation of the electroscalar field comprises a displacement charge (by analogy with the field created by Maxwell's displacement current), which leads to the necessity of taking into account both the field produced by these charges and the field of the charges themselves.

3) The time-dependent density of the electric charges accumulated along a longitudinal line enhances the longitudinal superposition of the charges. With the growth of the number of charges along the longitudinal line, the electroscalar field strength increases as well. On the contrary, the electromagnetic field generated by the field of charges incurs energy losses in the course of propagation and has no sources of replenishment.

4) In solid states the electroscalar radiation loses its energy in the cavities and due to that produces a corresponding plasmon electroscalar signal. The ability to detect the 
amplitude and frequency of this radiation would allow to observe the internal structure of the object.

5) The work done by the electroscalar field on a charged particle discloses the physical meaning of the negative energy of charged particles, shows the meaning of motion velocity for any charged particle and its ultimate value, as well as how the particle is transferred into the compound state by way of changing its electrical status. It should be noted that in the absence of an appropriate object to get into a compound state with, the particle can continue to lower it electrical status.

6) Decreasing the electrical status of the particle causes the particle to couple with any other body and make a transition into a compound state during the contact with an object. This state is the state of PHYSICAL VACUUM.

Thus, there is a possibility that the changes entailed by this new electroscalar dynamics are of profound nature and do not come into contradiction with those properties of the electromagnetic dynamics which make it convincing and, as a result, these properties can be included into the electrodynamics as a second dynamics of charge motion. The observation of neutrinos from the pp-cycle sheds light [12] on this fundamental problem. The electron, in particular, may be responsible for the high stability of atomic and molecular structures owing to the possible process of electromagnetic excitation of two electrons in an atom or molecule that exchange scalar photons, which leads to a strong coupling with the electron of the atom or molecule. Such processes may govern the confinement process in the atomic and molecular structures.

\section{Manifestations of the Electroscalar Properties in Nature}

The properties and potentialities of the electroscalar field find their ways of realization in nature and in the live bio-organism of this field which represents a new type of energy. Organisms and fishes with electrosensitivity and a capability to register the electroscalar radiation can be observed in nature [13]. Geophysical studies point to possible sources of this field at the Sun [14]. In biological investigations a great attention is paid to the organizing role of electric phenomena as well as to the possibility of controlling the growth and meaningfulness of the energy paradigm.

\section{Acknowledgements}

I thank the Editor and the referee for their remarks and comments. Also, I would like to thank Dr. V.B. Priezzhev for his attention and discussions. I am deeply obliged to my wife Liudmila for assistance in printing and supporting me.

\section{References}

[1] Zaimidoroga, O.A. (2016) Journal of Modern Physics, 7, 808-818. http://dx.doi.org/10.4236/jmp.2016.78074

[2] Zaimidoroga, O.A. and Podgainy, D.V. (2010) Observation of Electroscalar Radiation during an Eclipse of the Sun. Proceedings of International conference of the Astroparticle and 
Cosmic Rays Physics, Como, 1-7.

[3] Love, A.E.H. (1927) A Treatise on the Mathematical Theory of Elasticity. Cambridge University Press, Cambridge, UK.

[4] Landau, L.D. and Lifshitz, E.M. (1986) Theory of Elasticity, Vol. 7. 3rd Edition, Butterworth-Heinemann Publishing Limited.

[5] Podgainy, D.V. and Zaimidoroga, O.A. Nonrelativistic Theory of Electroscalar Field and Maxwell Electrodynamics. arXiv:1005.3130.

[6] Podgainy, D.V. and Zaimidoroga, O.A. Relativistic Dynamics of a Charged Particle in an Electroscalar Field. arXiv:1203.2490.

[7] Zeldovich, Ia.B. (1981) Uspehi Physicheskih Nauk, 133.

[8] Mostepanenko, A.M. and Mostepanenko, B.M. (1985) Nature, 3, 83.

[9] Wang, G.W., Sevick, E.M., Mittag, E., et al. (2002) Physical Review Letters, 89, Article ID: 050601. http://dx.doi.org/10.1103/PhysRevLett.89.050601

[10] Protsenko, I. and Rudoy, V.O. (2005) Plasmons resonanses in getero and nanostructures, Izvestia, 70, 514.

[11] Jackson, J.D. (1962) Classical Electrodynamics. Wiley, New York.

[12] Borexino Collboration (2014) Nature, 512, 383. http://dx.doi.org/10.1038/nature13702

[13] Bullock, T.H., Hopkins, C.D., Popper, A.N. and Fay, R.R. (2005) Electroreception Series: Springer Handbook of Auditory Research, Vol. 21, 472.

[14] Lavrentiev, M.M., Eganova, I.A., Luzet, M.K. and Fominyh, C.F. (1990) DAN, 314, 352.

\section{Submit or recommend next manuscript to SCIRP and we will provide best service} for you:

Accepting pre-submission inquiries through Email, Facebook, LinkedIn, Twitter, etc. A wide selection of journals (inclusive of 9 subjects, more than 200 journals)

Providing 24-hour high-quality service

User-friendly online submission system

Fair and swift peer-review system

Efficient typesetting and proofreading procedure

Display of the result of downloads and visits, as well as the number of cited articles

Maximum dissemination of your research work

Submit your manuscript at: http://papersubmission.scirp.org/

Or contact jmp@scirp.org 